\title{
Endothelial progenitor cells attenuate the lung ischemia/ reperfusion injury following lung transplantation via the endothelial nitric oxide synthase pathway
}

Wei Gao, MD, Tao Jiang, MD, Yan-hong Liu, MD, Wen-gang Ding, MD, Chang-chun Guo, MD, and Xiao-guang Cui, MD

\section{ABSTRACT}

Objective: Endothelial progenitor cells (EPCs) can improve endothelial integrity. This study aimed to examine the effects and the mechanism of EPCs on lung ischemia-reperfusion injury (LIRI).

Methods: Wistar rats were randomized into the sham or the left lung transplantation group. The recipients were randomized and treated with vehicle as the LIRI group, with EPC as the EPC group, or with N5-(1-iminoethyl)-1-ornithine-pretreated EPC as the EPC/L group ( $n=8$ per group). The ratios of arterial oxygen partial pressure to fractional inspiratory oxygen were measured. The lung wet-to-dry weight ratios, protein levels, and injury, as well as the levels of plasma cytokines, were examined. The levels of endothelin (ET)-1, endothelial nitric oxide synthase (eNOS), phosphorylated eNOS, inducible NOS, phosphorylated myosin light chain, nuclear factor- $\kappa \mathrm{Bp} 65$, Bax, Bcl-2, cleaved caspase-3, and myeloperoxidase in the graft lungs were detected.

Results: Compared with the LIRI group, EPC treatment significantly increased the ratios of arterial oxygen partial pressure to fractional inspiratory oxygen and decreased the lung wet-to-dry weight ratios and protein levels in the grafts, accompanied by increasing eNOS expression and phosphorylation, but decreasing endothelin-1, inducible NOS, phosphorylated nuclear factor-kBp65, phosphorylated myosin light chain expression, and myeloperoxidase activity. EPCs reduced lung tissue damage and apoptosis associated with decreased levels of Bax and cleaved caspase- 3 expression, but increased Bcl-2 expression. EPC treatment significantly reduced the levels of serum proinflammatory factors, but elevated levels of interleukin-10. In contrast, the protective effect of EPCs were mitigated and abrogated by N5-(1-iminoethyl)-1-ornithine pretreatment.

Conclusions: Data indicated that EPC ameliorated LIRI by increasing eNOS expression. (J Thorac Cardiovasc Surg 2019;157:803-14)

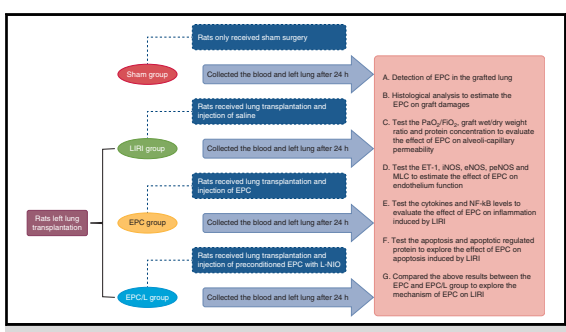

EPCs significantly reduce the lung injury induced by ischemia reperfusion.

Central Message

EPCs attenuate the LIRI associated with mitigating injury to the endothelium and attenuating NF- $\kappa \mathrm{B}$ signaling, inflammation, and apoptosis via eNOS.

\section{Perspective}

About $20 \%$ to $35 \%$ of recipients develop lung ischemia-reperfusion injury (LIRI) after lung transplantation. Endothelium injury plays a key role in LIRI. Endothelial progenitor cells (EPCs) can ameliorate endothelium injury, inhibit inflammation, and reduce the LIRI after lung transplantation. The protective effect of EPCs on LIRI is mainly mediated by enhancing eNOS signaling.

See Editorial Commentary page 815
Lung transplantation is the only treatment for patient with end-stage lung disease. However, about $20 \%$ to $35 \%$ of recipients develop lung ischemia-reperfusion injury (LIRI), which is a major risk factor for the mortality of recipients

\footnotetext{
From the Department of Anesthesiology, the Second Affiliated Hospital of the Harbin Medical University, Harbin, Heilongjiang Province, China.

Supported by a grant from the National Natural Science Foundation of China (No. 81500074) and Heilongjiang Natural Science Foundation (No. QC2017112).

Received for publication Jan 24, 2018; revisions received July 28, 2018; accepted for publication Aug 11, 2018; available ahead of print Nov 1, 2018.

Address for reprints: Xiao-guang Cui, MD, Department of Anesthesiology, the Second Affiliated Hospital of the Harbin Medical University, 246 Xuefu Rd, Harbin 150081, Heilongjiang Province, China (E-mail: cuixiaoguang66@163.com). 0022-5223/\$36.00

Copyright (c) 2018 by The American Association for Thoracic Surgery https://doi.org/10.1016/j.jtcvs.2018.08.092
}

within the first 90 days postoperation. ${ }^{2}$ Moreover, LIRI can increase the risk of immune rejection and pulmonary fibrosis. ${ }^{3}$ Endothelium injury is crucial for the development of LIRI. During the process of LIRI, hypoxemia and reoxygenation can impair the endothelium and increase alveoli-capillary permeability leading to lung injury. ${ }^{4,5}$

$\square$ Scanning this QR code will take you to the article title page to access supplementary information. 


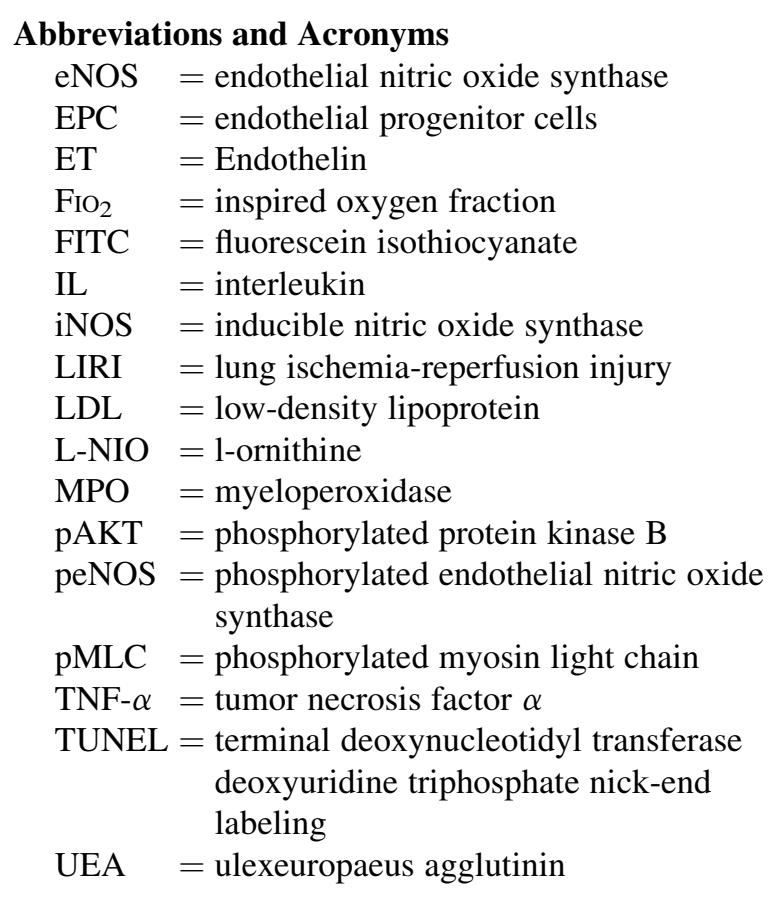

Hence, protection of the endothelium is significant in management of patients undergoing lung transplantation.

Endothelial progenitor cells (EPCs) can promote vascular regeneration and inhibit inflammation in acute lung injury, ${ }^{6}$ myocardial ischemia-reperfusion injury, ${ }^{7}$ and vascular transplantation. ${ }^{8}$ EPCs can improve endothelial dysfunction and preserve integrity of the alveolarcapillary barrier ${ }^{6}$ to enhance the survival of individuals with acute lung injury. ${ }^{9}$ EPCs can also modulate inflammation by decreasing proinflammatory cytokine production but increasing anti-inflammatory cytokine production. ${ }^{6,10,11}$ The precise mechanisms underlying the action of EPCs in enhancing the function of lung grafts are still unknown. Recent studies have shown that endothelial nitric oxide synthase (eNOS) is crucial for the function of EPCs $^{12,13}$ and can promote the attachment, migration, and tube formation of EPCs. ${ }^{14}$ However, whether EPCs can modulate the LIRI and what role eNOS plays in the process of LIRI have not been clarified. We hypothesized that EPCs may improve graft lung function through amelioration of LIRI. In this study, we employed a rat model of lung transplantation to determine the effect of EPCs or eNOS inhibitor-treated EPCs on LIRI and to explore the possible mechanisms underlying the action of EPC on LIRI.

\section{MATERIALS AND METHODS Animals}

Male Wistar rats weighing about 200 to $250 \mathrm{~g}$ were obtained from the animal care facility of Harbin Medical University and housed in a specific pathogen-free facility with free access to food and water. This study was carried out in accordance with the national guidelines for animal studies and approved by the Institutional Animal Care and Use Committee of the Second Affiliated Hospital of Harbin Medical University.

\section{Isolation and Culture of EPCs}

Isolation and culture of EPCs were performed as described previously. ${ }^{6}$ Briefly, peripheral blood mononuclear cells were isolated from Wistar rats by density gradient centrifugation using the Ficoll-Plaque Plus (Amersham Pharmacia Biotech, Uppsala, Sweden). The mononuclear cells were cultured in endothelial growth medium-2 (Lonza, Basel, Switzerland) containing 2\% of fetal bovine serum at $37^{\circ} \mathrm{C}, 5 \%$ carbon dioxide in 6-well plates that had been coated with human fibronectin. The cells were exposed to fresh medium daily and cultured for 10 days. The adherent cells (known as early EPCs) were harvested by trypsinization and used for characterization.

\section{Characterization of EPCs}

EPCs (approximately $24 \times 10^{4}$ cells/well) were treated in triplicate with $30 \mu \mathrm{g} / \mathrm{mL}$ DiI-acetyl-low-density lipoprotein (LDL) (Invitrogen, Carlsbad, Calif) for 4 hours and $50 \mu \mathrm{g} / \mathrm{mL}$ fluorescein isothiocyanate (FITC)-conjugated ulexeuropaeus agglutinin-1 (UEA-1) (Sigma-Aldrich, Saint Louis, Mo) for 2 hours. The EPCs were examined under a fluorescent confocal microscope. The cells with dual green UEA-1 and red DiI-labeled acetyl-LDL staining were identified as differentiating EPCs. Furthermore, the cells were stained with fluorescent anti-vascular endothelial growth factor receptor 2 (Abcam, Cambridge, United Kingdom) and anti-CD34 (Santa Cruz Biotechnology, Santa Cruz, Calif) and examined by microscopy. Moreover, the cells were stained with FITC-anti-CD14 and phycoerythrin-anti-CD45 and the percentages of CD14+CD45+ early EPCs and CD14-CD45- advanced EPCs were characterized by flow cytometry. Finally, some EPCs were incubated with a specific eNOS inhibitor, N5-(1-iminoethyl)-1-ornithine (L-NIO) $10 \mu \mathrm{M}$ (Santa Cruz Biotechnology) for 1 hour before treatment of rats.

\section{Lung Transplantation}

The rat left lung transplantation was performed, as described previously (Video 1). ${ }^{15}$ Briefly, the rats were food-fasted for 12 hours and randomized into the sham, LIRI, EPC, and EPC/L-NIO (EPC/L) groups ( 8 rats per group) by a computer-generated random number table. The rats were injected intraperitoneally with $3 \%$ pentobarbital sodium $(30 \mathrm{mg} / \mathrm{kg})$. The donor rats were intubated with 12-gauge catheter that connected to a Harvard Rodent Ventilator (Model 683; Harvard Apparatus, Boston, Mass) and ventilated with a tidal volume of $10 \mathrm{~mL} / \mathrm{kg}$ and respiratory rate of 50 breaths/minute, which led to an inspired oxygen fraction $\left(\mathrm{FiO}_{2}\right)$ of $50 \%$ (50\% oxygen and $50 \%$ nitrogen) at positive end-expiratory pressure of $2 \mathrm{cmH}_{2} \mathrm{O}$ and inspiratory expiratory ratio of 1 to 1 . After heparinization, pulmonary arteries were injected with $20 \mathrm{~mL}$ cold saline at $20 \mathrm{cmH}_{2} \mathrm{O}$ pressure. Hearts and lungs

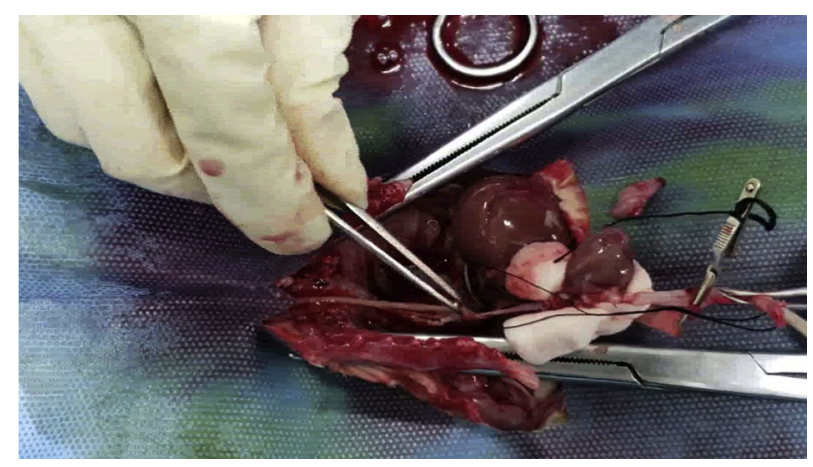

VIDEO 1. Procedure of lung transplantation. Video available at: https:// www.jtcvs.org/article/S0022-5223(18)32487-5/fulltext. 
were harvested after inflating the lungs at end-tidal volume. The left lung arterial and left venous blood vessels, and bronchial tubes were cropped and attached to a cuff tube and preserved in saline at $4^{\circ} \mathrm{C}$ for 90 minutes. ${ }^{16}$

The recipient rats were anesthetized and ventilated in the same way as the donor rats. After being intubated with a 12-gauge catheter, the right femoral artery and veins of individual recipients were cannulated to monitor blood pressure and allow injection with saline $(10 \mathrm{~mL} / \mathrm{kg} / \mathrm{h})$. After thoracotomy, the left main bronchus, pulmonary artery, and vein were exposed and dissociated. Subsequently, the left bronchus, artery, and veins of recipients were collapsed and removed, and then anastomosed with a donor lung by the Cuff technique. ${ }^{15}$ During the lung transplantation, the tidal volumes were changed to $6 \mathrm{~mL} / \mathrm{kg}$ and restored to $10 \mathrm{~mL} / \mathrm{kg}$ immediately after reperfusion. Immediately after reperfusion following the lung transplantation, the recipients were injected intravenously with $1 \mathrm{~mL}$ phosphate buffered saline (the LIRI group), approximately $10^{6}$ DiI-acetyl-LDL-labeled EPC in $1 \mathrm{~mL}$ phosphate buffered saline (the EPC group), ${ }^{6}$ or the same number of L-NIO treated EPCs (EPC/L group). The sham group of rats underwent a sham surgery and were injected with phosphate buffered saline. During the procedure, the body temperature of individual rats was measured by a rectal thermometer and maintained between $37^{\circ} \mathrm{C}$ and $39^{\circ} \mathrm{C}$. After the chest was closed and spontaneous respiration was restored, the recipients were extubated. The recipients were treated with $0.125 \%$ ropivacaine by local infiltration analgesia every 12 hours.

At baseline and 24 hours after reperfusion, their arterial blood gases were analyzed and their peripheral blood samples were collected to prepare serum samples. At 24 hours after reperfusion, all rats were killed and lung grafts were collected. The left upper part of the lung graft was stored at $-80^{\circ} \mathrm{C}$ and the remaining lung grafts were fixed in $4 \%$ paraformaldehyde for histology analysis.

\section{Arterial Blood Gas Analysis}

The arterial blood gases were analyzed using a Bayer Rapidlab 348 (Bayer Diagnostics, Leverkusen, Germany), and the $\mathrm{PAO}_{2} / \mathrm{FIO}_{2}$ ratios were calculated.

\section{Alveoli-Capillary Permeability in the Lung Grafts}

The left upper part of the lung graft in individual recipients was collected and wet-weighed, then dried at $60^{\circ} \mathrm{C}$ for 48 hours. The ratios of wet/dry weights of individual graft samples were calculated. The protein concentrations in the transplanted lung homogenates were measured using the Bradford method.

\section{Measurement of Serum Cytokines}

The levels of serum tumor necrosis factor (TNF)- $\alpha$, interleukin (IL)- $1 \beta$, IL-6, IL-8, and IL-10 in individual rats were determined by enzyme-linked immunosorbent assay (Wuhan Boster Bio-Engineering, Wuhan, China), according to the manufacturers' instructions. The limitation of detection is $2 \mathrm{pg} / \mathrm{mL}, 2 \mathrm{pg} / \mathrm{mL}, 5 \mathrm{pg} / \mathrm{mL}, 2 \mathrm{pg} / \mathrm{mL}$, and $2 \mathrm{pg} / \mathrm{mL}$ for TNF- $\alpha$, IL-1 $\beta$, IL-6, IL-8, and IL-10, respectively. Moreover, the activity of myeloperoxidase (MPO) in the transplanted lung tissues was detected with a radioimmunoassay kit (Nanjing Jiancheng, Nanjing, China).

\section{Histopathology Examination}

The left lower parts of lung graft tissues were fixed in $4 \%$ paraformaldehyde and paraffin-embedded. The tissue sections $(4 \mu \mathrm{m})$ were stained with hematoxylin and eosin. The lung injury was evaluated for alveolar congestion, edema, hemorrhage, neutrophil infiltration, the alveolar wall thickness, and hyaline membrane formation by 2 pathologists in a blinded manner.

\section{Detection of EPCs in the Grafted Lungs}

To examine whether EPCs could migrate into the lung grafts, EPCs (approximately $10^{6}$ cells $/ \mathrm{mL}$ ) were labeled with $(30 \mu \mathrm{g} / \mathrm{mL}$ )
DiI-acetyl-LDL at $37^{\circ} \mathrm{C}$ for 2 hours and injected into individual recipients. Twenty-four hours postinjection, the rats were killed and their lung grafts were fixed with $4 \%$ paraformaldehyde and paraffin-embedded. The graft tissue sections $(5 \mu \mathrm{m})$ were stained with 4,6-diamidino-2-phenylindole and examined under a fluorescent microscope.

\section{Apoptosis of Lung Tissue}

The severity of endothelial cell apoptosis in the lung grafts was determined by terminal deoxynucleotidyl transferase deoxyuridine triphosphate nick-end labeling (TUNEL) assay (Roche Diagnostics $\mathrm{GmbH}$, Science, Mannheim, Germany). Briefly, the lung graft tissue sections $(4 \mu \mathrm{m})$ were treated with proteinase $\mathrm{K}$ at $37^{\circ} \mathrm{C}$ for 30 minutes, and immersed in TUNEL reaction mixture (terminal deoxynucleotidyl transferase and fluorochrome-conjugated deoxyuridine triphosphate) at $37^{\circ} \mathrm{C}$ for 60 minutes in darkness. After being washed, the sections were counterstained with $1 \mu \mathrm{g} / \mathrm{mL}$ 4,6-diamidino-2-phenylindole. The fluorescent signals were observed under a fluorescence confocal microscope.

\section{Western Blotting}

Lung grafts was homogenized and lyzed in lysis buffer containing proteinase inhibitors (Beyotime Biotechnology, Shanghai, China). After being centrifuged, the protein concentrations of individual samples were determined by the Bradford assay. The tissue lysates ( $30 \mu \mathrm{g} / \mathrm{lane})$ were separated by sodium dodecyl sulfate polyacrylamide gel electrophoresis on $12 \%$ gels and transferred onto polyvinylidene fluoride membranes. The membrane was blocked with $5 \%$ fat-free dry milk in tris-buffer solution Tween, and incubated with antibodies against phosphorylated protein kinase B (p-AKT), endothelin (ET)-1, eNOS, phosphorylated eNOS (p-eNOS), inducible NOS (iNOS), phosphorylated myosin light chain (pMLC) (pSer18, Sigma Aldrich), phosphorylated nuclear factor $\kappa \mathrm{Bp} 65$ (Santa Cruz Biotechnology), Bax, Bcl-2, and cleaved caspase-3 (Cell Signaling Technology, Danvers, Mass). The bound antibodies were detected with horseradish peroxidase-conjugated secondary antibodies (Santa Cruz Biotechnology) and visualized using the enhanced chemiluminescence reagents. The relative levels of target proteins to the control $\beta$-actin were determined by densitometric analysis using Image J software (National Institutes of Health, Bethesda, Md).

\section{Statistical Analysis}

Data were analyzed using a repeated measure analysis of variance, with 2 levels by time (pre- and posttest) and considering groups as intersubjects factor. When differences were noted, Bonferroni post hoc analysis was performed to identify the source of the difference.

The nonnormal distribution data are presented by median and interquartile range plots and analyzed with Mann-Whitney $U$ test, and the $P$ value was adjusted with Bonferroni. All data were analyzed using the IBM-SPSS Statistics version 19.0 (IBM-SPSS Inc, Armonk, NY).

\section{RESULTS}

\section{Characterization of EPCs}

After culture for 10 days, the potential EPCs were grown on plates with typical spindle shape (Figure 1, $A$ ) and were positive for anti-vascular endothelial growth factor receptor 2 and anti-CD34 staining (Figure $1, B$ and $C$ ). Furthermore, after treatment with FITC-labeled UEA-1 and DiI-acetyl-LDL, almost all of the cells displayed fluorescent signals of FITC and DiL, indicative of UEA-1 and LDL uptake, which is a hallmark of differentiating EPCs (Figure 1, $D-F$ ). Further flow cytometry analysis indicated that the percentages of early CD14+CD $45+$ and advanced CD14-CD45- EPCs were about 9.8\% $\pm 4.1 \%$ and $25.9 \% \pm 9.9 \%$, respectively. 

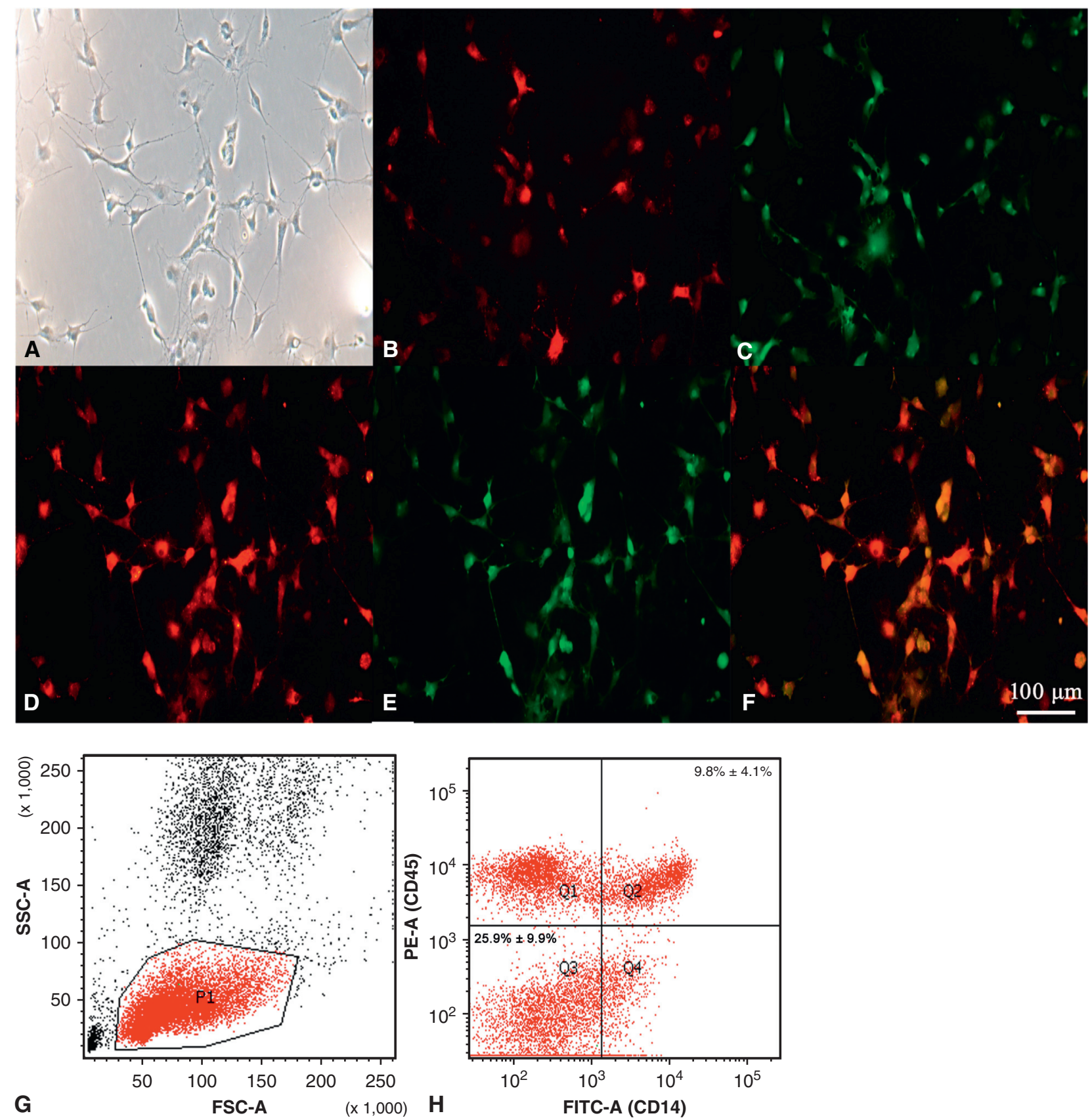

FIGURE 1. Characterization of endothelial progenitor cells (EPCs) in vitro. A, Peripheral blood mononuclear cells were isolated from rats and cultured in $2 \%$ fetal bovine serum endothelial growth medium- 2 for 10 days. The cells were examined under a light microscope ( $400 \times$ magnification). B, Cells stained with phycoerythrin-antivascular endothelial growth factor receptor 2 examined under a fluorescent microscope. C, Cells stained with fluorescein isothiocyanate (FITC)-anti-CD34 examined under a fluorescent microscope. D, Cells labeled with DiI-acetyl-low-density lipoprotein examined under a fluorescent microscope. E, Cells labeled with FITC-ulexeuropaeus agglutinin-1 examined under a fluorescent microscope. F, The merged image of panels D and E. G, Cells further stained with FITC-anti-CD14. H, Cells further stained with phycoerythrin-anti-CD45. The percentages of CD14-CD45- and $\mathrm{CD} 14+\mathrm{CD} 45+$ cells were $25.9 \% \pm 9.9 \%$ and $9.8 \% \pm 4.1 \%$, respectively. Data are representative images (400× magnification) from 3 separate experiments. SSC-A, Side scatter A; FSC-A, forward scatter A; PE-A, phycoerythrin A; FITC-A, fluorescein isothiocyanate A.

\section{Detection of EPCs in the Transplanted Lung}

Individual rats in the EPC and $\mathrm{EPC} / \mathrm{L}$ group were injected intravenously with about $1 \times 10^{6} \mathrm{EPC}$ or
L-NIO-treated EPCs that had been labeled with DiIacetyl-LDL, respectively. The transplanted lung sections were prepared from individual groups of rats. There 

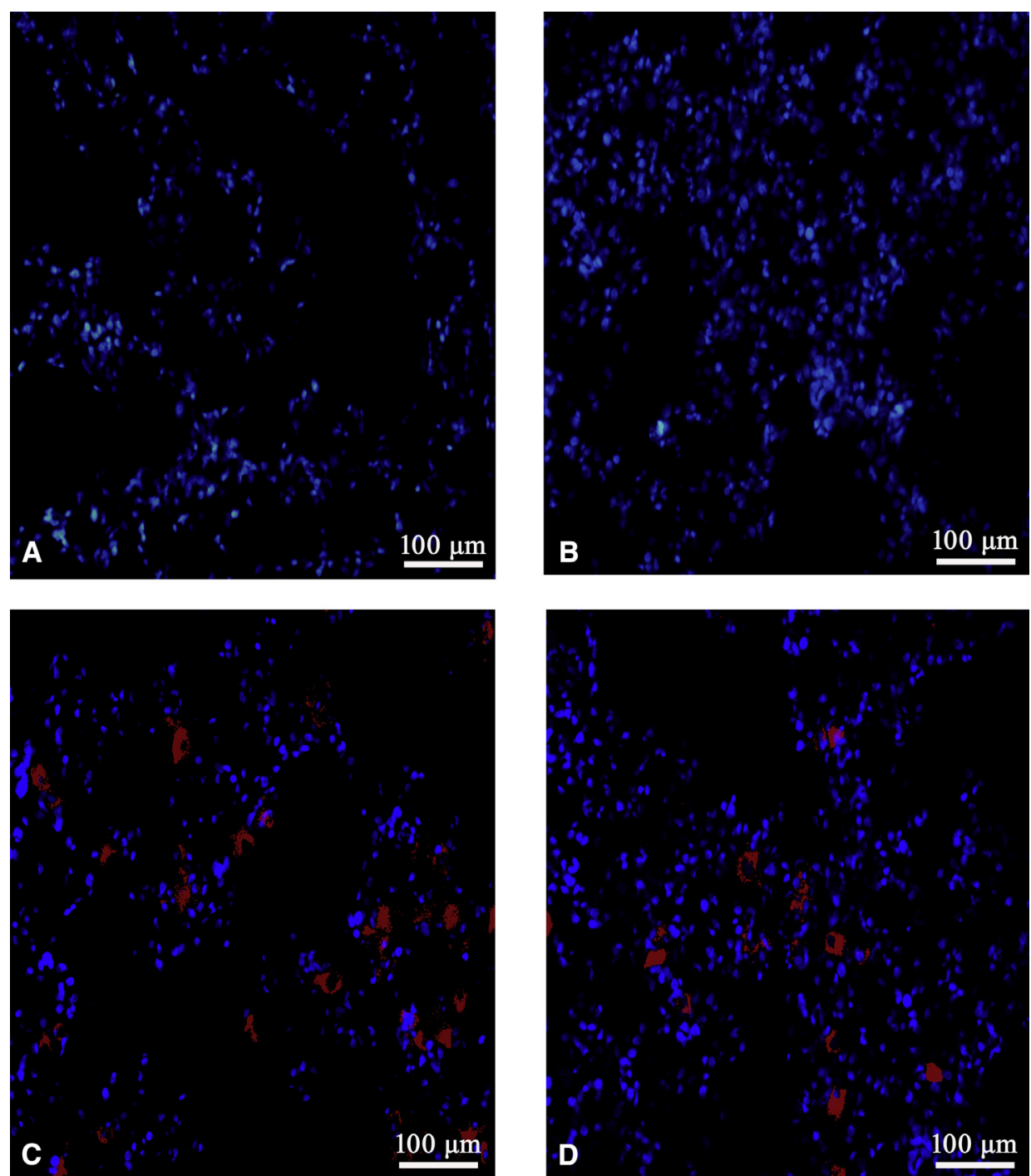

FIGURE 2. Detection of endothelial progenitor cells (EPCs) in the grafted lung. EPC and 1-ornithine-treated (appropriately $10^{6}$ cells $/ \mathrm{mL}$ ) were labeled with DiI-acetyl-low-density lipoprotein for 2 hours. Following lung transplantation, the recipients were injected intravenously with the DiI-acetyl-low-density lipoprotein-labeled EPC or 1-ornithine-treated EPCs (appropriately $10^{6}$ cells per rat) immediately after reperfusion. The sham and lung ischemia-reperfusion injury groups of rats did not receive EPC injection. Twenty-four hours after injection, the lung graft sections ( $5 \mu \mathrm{m})$ were stained with 4,6-diamidino-2-phenylindole. The cells were examined under a fluorescent confocal microscope. Data are representative images (magnification $\times 200)$ from each group $(n=8$ per group). A, There was no detectable EPC in the sham group. B, There was no detectable EPC in the lung ischemia-reperfusion injury group of rats. C, EPCs are present in the lung grafts of the EPC rats. D, EPCs are present in the lung grafts of 1-ornithinepretreated EPC $(\mathrm{EPC} / \mathrm{L})$ rats. The frequency of 1-ornithine-treated EPCs in the transplanted lung was significantly lower than that of the EPC group $(5.5 \% \pm 1.6 \%$ vs $11.8 \% \pm 2.5 \% ; P=.0001$, determined by Student $t$ test).

were no detectable EPCs in the sham group (Figure 2, A) and LIRI group (Figure 2, $B$ ) of rats that had not been injected with any EPC. The EPCs were detected in the lung grafts of the EPC (Figure 2,C) and EPC/L (Figure 2, D) groups of rats. Quantitative analysis indicated the percentages of EPCs in the lung grafts of the EPC/L group were significantly lower than that in the EPC group $(5.5 \% \pm 1.6 \%$ vs $11.8 \% \pm 2.5 \% ; P<.05)$. Such data indicated the infused EPCs effectively migrated into the lung grafts, partly dependent on the eNOS signaling in rats.

\section{EPCs Reduce LIRI in the Lung Grafts of Rats}

Pathologic examination indicated the typical pathologic changes in the lung grafts in the LIRI, EPC, and EPC/L groups, compared with the sham group ( $\mathrm{n}=8$ per group). There were lung edema, increased alveolar wall thickness, alveolar damage, hyaline membrane formation, and 


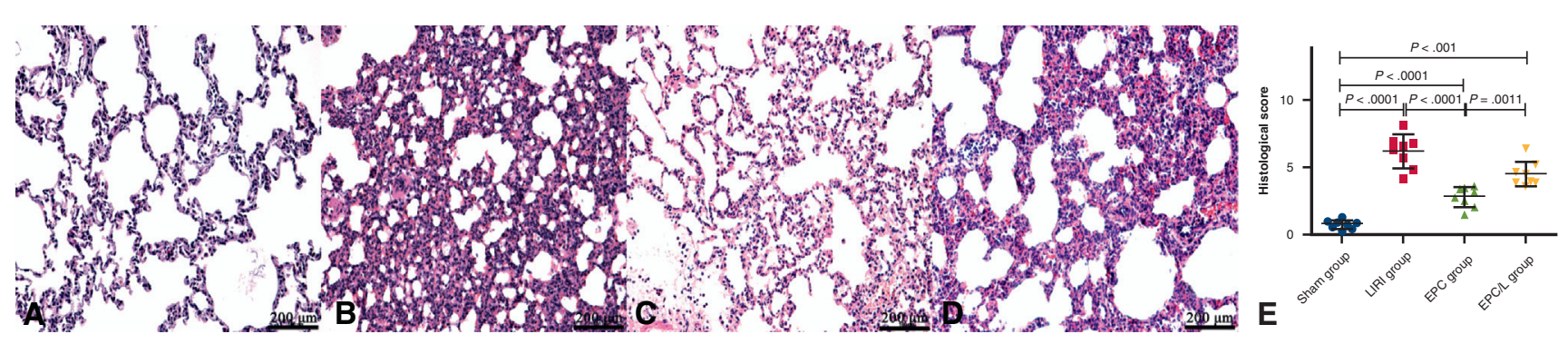

FIGURE 3. Endothelial progenitor cells $(E P C s)$ mitigate the ischemia-reperfusion-induced damage and inflammation in the grafted lungs of rats. The grafted lung tissue sections were stained with hemotoxylin and eosin and examined under a light microscope. Data are representative images (magnification $\times 200)$ or expressed as the individual mean value from each group of rats $(\mathrm{n}=8$ per group). A, The sham group. B, The lung ischemia-reperfusion injury (LIRI) group. C, The EPC group. D, The 1-ornithine-pretreated EPC (EPC/L) group. E, The quantitative analysis. ( sham group, $\square$ LIRI group, $\triangle$ EPC group, $\nabla$ and EPC/L group.) The upper and lower borders of the box represent the standard deviation. The middle horizontal line represents the mean.

hemorrhage accompanied by inflammatory infiltrates in the lung grafts (Figure 3). The lung injury scores in the EPC group were less than that in the LIRI group $(P<.001)$ and this improvement was reduced in the EPC/L group $(P=.002)$.

\section{EPCs Improve Alveoli-Capillary Permeability}

Measurement of the lung function revealed that the $\mathrm{PAO}_{2} / \mathrm{FIO}_{2}$ ratios in the lung transplantation groups of rats were less than that in the sham control group rats (all $P$ values $<.001)$, indicating that the lung transplant-related ischemia-reperfusion impaired pulmonary function in rats (Figure $4, A$ ). Furthermore, the $\mathrm{PAO}_{2} / \mathrm{FIO}_{2}$ ratio in the EPC group of rats was significantly higher than that in the LIRI group, and significantly reduced in the EPC/L group $(P$ value $<.001$ for all $)$ In addition, the arterial blood samples and transplanted lung tissues of 8 rats in each group were collected to evaluate the effect of EPCs on alveolicapillary permeability. The lung wet/dry weight ratios and protein levels in the EPC group were significantly less than that in the LIRI group $(P<.001)$ (Figure $4, B$ and $C$ ) and the improvement of wet/dry weight ratios $(P=.001)$ and protein levels $(P<.001)$ were significantly mitigated in the $\mathrm{EPC} / \mathrm{L}$ group.

\section{EPCs Reduce Endothelial Cell Injury}

The expression of p-AKT, ET-1, iNOS, eNOS, peNOS, and pMLC to control $\beta$-actin in individual groups of transplanted lung graft tissues were determined by Western blot (Figure 5, $A)(\mathrm{n}=8$ per group). Compared with the LIRI group, EPC significantly mitigated the ischemiareperfusion-upregulated ET-1, pMLC, and iNOS expression, but enhanced p-AKT, eNOS, and peNOS expression in the lung grafts $(P<.001$ for all except $P=.022$ for eNOS) (Figure $5, B$ ). These changes induced by EPCs were significantly reduced in the $\mathrm{EPC} / \mathrm{L}$ group $(P=.013$ for ET-1, $P=.002$ for eNOS, $P<.001$ for peNOS, and $P=.001$ for iNOS and pMLC).

\section{EPC Inhibits Inflammation}

The effect of EPCs on inflammation in the lung grafts was determined in individual groups ( $\mathrm{n}=8$ per group). In comparison with the sham group, the levels of serum cytokines and MPO activity significantly increased in the recipient rats $(P$ values $<.001$ for all) (Figure $6, A)$. EPCs significantly reduced the levels of serum TNF- $\alpha$, IL- $1 \beta$, IL-6, and IL-8, but elevated IL-10 in rats, relative to the LIRI group $(P<.001$ for TNF- $\alpha, P=.002$ for IL- $1 \beta$, $P<.001$ for IL-6 and IL-8, and $P=.01$ for IL-10). The immune-regulatory effects of EPCs were significantly reduced in the EPC/L group $(P=.002$ for TNF- $\alpha$, $P=.04$ for IL-1 $\beta, P=.03$ for IL-6 and IL- 8 , and $P=.01$ for IL-10). Similarly, the activity of MPO and levels of phosphorylated nuclear factor-kBp65 in the lung grafts of the EPC group were significantly lower than that in the LIRI and EPC/L groups $(P=.025$ and $P<.001$, respectively) (Figure 6, $A$ and $B$ ), but remained significantly higher than that in the sham group $(P<.001$ for both $)$.

\section{EPCs Attenuate Apoptosis}

The effect of EPCs on apoptosis in lung grafts of individual groups ( $\mathrm{n}=8$ per group) was determined by TUNEL assay. There were no detectable apoptotic cells in the sham group (Figure $7, A$ ), whereas significantly increased frequency of apoptotic cells was detected in the lung graft tissues of the LIRI group $(P<.001)$ (Figure $7, B$ and $E$ ). The percentages of apoptotic cells in the lung grafts of the EPC group were significantly lower than that in the LIRI group $(P<.001)$ (Figure 7, $C$ and $E$ ), but the antiapoptotic effect of EPCs was mitigated in the EPC-L group $(P=.005)$ (Figure 7, $D$ and $E$ ). Western blot analyses indicated that the relative levels of Bax, Bcl-2, and cleaved caspase-3 expression in the lung grafts of the LIRI group were significantly higher than that in the sham group $(P$ values $<.001$ for all) (Figure 8$)$, whereas significantly decreased levels of Bax and cleaved caspase- 3 but increased Bcl-2 were detected in the lung grafts of the EPC group $(P=.003, P<.001$, and $P<.001)$. However, the 


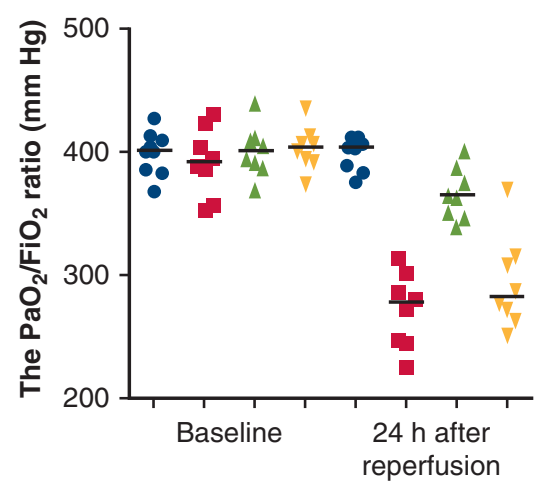

A

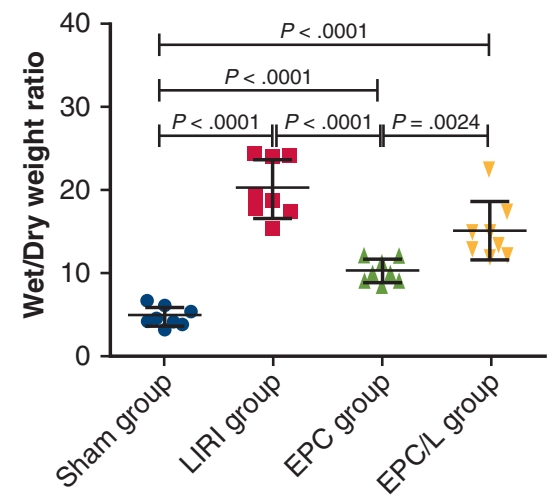

B

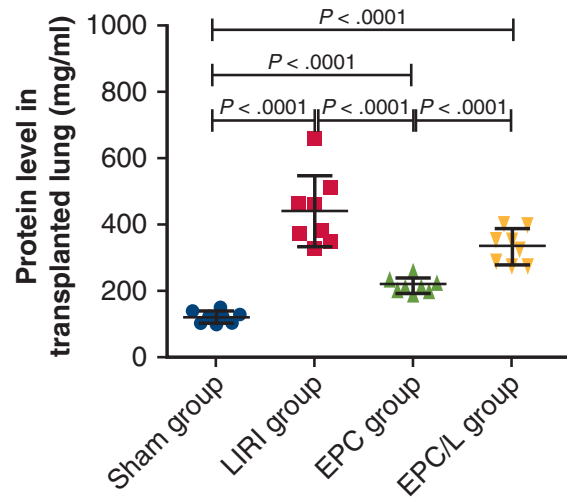

C

FIGURE 4. Endothelial progenitor cells $(E P C s)$ improve the function of grafted lung in rats. The ratio of arterial oxygen partial pressure to inspired oxygen fraction $\left(\mathrm{PAO}_{2} / \mathrm{FIO}_{2}\right.$ ratio $)$ in the sham, lung ischemia-reperfusion injury (LIRI), EPC, and l-ornithine-pretreated EPC $(E P C / L)$ groups of rats were measured before lung transplantation and 24 hours after treatment with vehicle phosphate buffered saline (LIRI group), EPC, or 1-ornithine-pretreated EPC. After being killed, the ratios of wet to dry weights of the grafted lungs and the levels of total proteins in the grafted lungs of individual groups of rats were determined. Data are expressed as the median (interquartile range) of individual groups ( $\mathrm{n}=8$ per group). A, The $\mathrm{PaO}_{2} / \mathrm{FiO}_{2} \mathrm{ratio}$. $\mathrm{B}$, The wet/dry weight ratio. C, The protein levels in transplanted lung tissue. ( sham group, $\square$ LIRI group, $\triangle$ EPC group, $\nabla$ and EPC/L group.) The upper and lower borders of the box represent the standard deviation. The middle horizontal line represents the mean.

antiapoptotic effect of EPCs was significantly reduced in the EPC/L group $(P=.005, P=.005$, and $P=.01)$.

\section{DISCUSSION}

EPCs can differentiate into endothelial cells and incorporate into the sites of injured vessels. ${ }^{17}$ Currently, there are 2 subtypes of EPCs with different vasculogenesis effects referred to as early EPCs and advanced EPCs. ${ }^{18}$ The early EPCs usually appear after 4 to 7 days of culture and they have low proliferation potency but can secrete angiogenic cytokines to improve angiogenesis. ${ }^{19}$ In contrast, the advanced EPCs have high proliferation potency and participate in neovasculogenesis. ${ }^{18}$ More importantly, a mixed population of early and advanced EPCs can regulate immune response, inflammation, and endothelial repair. ${ }^{6,20,21}$ Therefore, we investigated the effect of a mixed population of early and advanced EPCs on LIRI following lung transplantation.

In this study, we found that the isolated EPCs displayed unique characteristics and effectively migrated into the lung graft tissues, consistent with a previous report. ${ }^{22}$ Evidentially, EPC treatment significantly mitigated the wet/dry weight ratios, protein levels, pathologic changes, serum cytokine levels, and apoptotic cell percentages in the lung grafts, leading to improved pulmonary function. These indicate that EPC treatment reduces the alveolicapillary permeability, lung edema, and injury. ${ }^{15}$ That EPCs can improve endothelial integrity and inhibit inflammation support the notion that dysfunction and injury of the endothelium are crucial for the early development of LIRI. $^{23}$ Our novel findings suggest that EPC treatment may be valuable for management of LIRI. We are interested in further investigating whether EPC can incorporate into the endothelium to repair and maintain the integrity of the endothelium in lung grafts.

It is well known that ischemia-reperfusion induces endothelial cell injury and that injured endothelial cells can release ET-1, eNOS, and iNOS that promote the endothelial injury via strong microvascular constriction and nitric oxide and reactive oxygen species production. In contrast to injurous effect of iNOS, eNOS can reduce the expression of adhesion molecules. In the present study, we found significantly higher levels of ET-1, eNOS, and iNOS expression, eNOS, and MLC phosphorylation in the lung grafts, demonstrating endothelial injury following lung transplantation. $^{22,24}$ The higher levels of ET-1 may contribute to high levels of iNOS because ET-1 can promote iNOS expression. ${ }^{25}$ The higher levels of iNOS expression should enhance the production of nitric oxide, reactive oxygen species, and inflammatory cytokines, leading to acute inflammation. ${ }^{26}$ In contrast, the eNOS can protect from reperfusion-induced lung injury probably by inhibition of endothelial adhesion molecule expression. ${ }^{27} \mathrm{We}$ found that EPCs reduced the levels of ET-1 and iNOS expression, but upregulated eNOS expression and phosphorylation in the lung grafts, which may contribute to protective effect of EPCs. EPCs can improve the alveolar capillary hyperpermeability in the transplanted lung by protection of endothelial cells that may be attributed to downregulation of ET-1 and iNOS and upregulation of eNOS expression and phosphorylation. eNOS is downstream of the PI3K/AKT signal pathway. To explore 

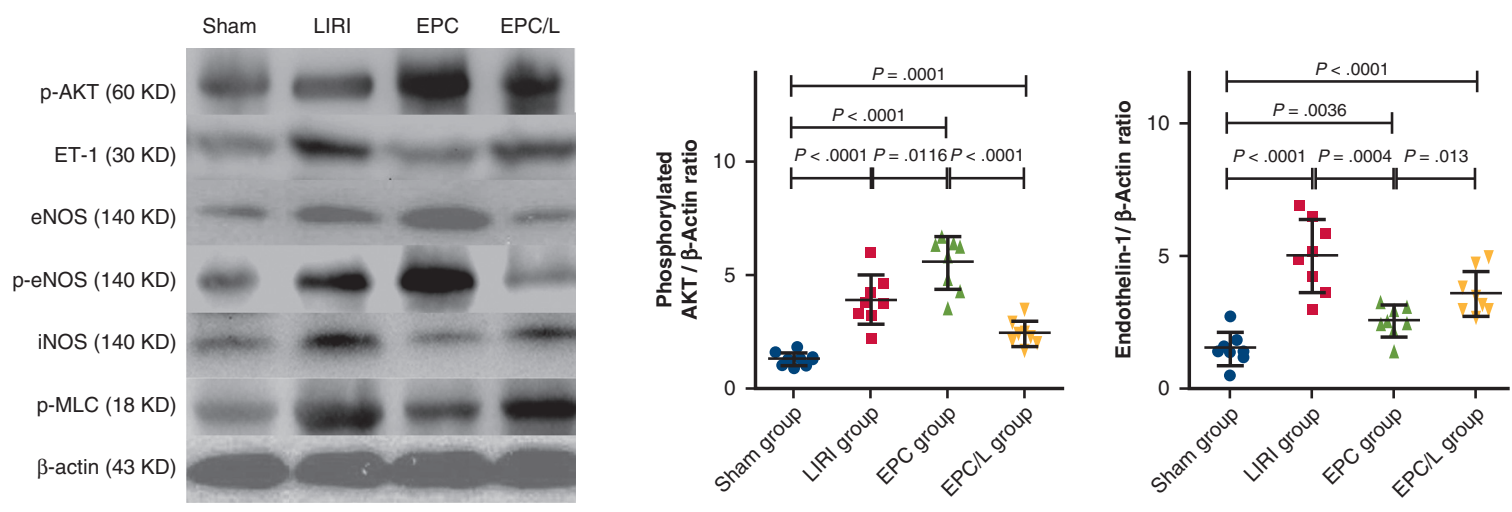

A
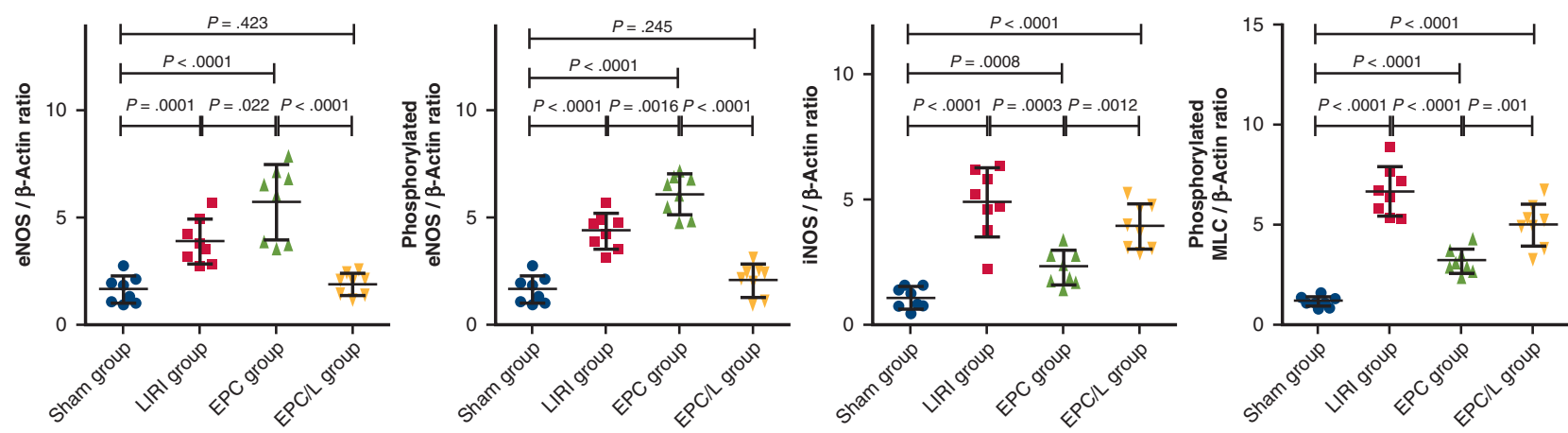

B

FIGURE 5. Endothelial progenitor cells $(E P C s)$ mitigate the ischemia-reperfusion-induced endothelium injury in grafted lungs. After treatment with vehicle phosphate buffered saline (lung ischemia-reperfusion injury [LIRI] group), EPC, or N5-(1-iminoethyl)-1-ornithine-treated EPC for 24 hours, the relative levels of endothelin-1 (ET-1), inducible nitric oxide synthase (iNOS), endothelial NOS (eNOS), phosphorylated eNOS, and myosin light chain to the control $\beta$-actin in the grafted lungs of individual groups of rats were determined by Western blot. Data are representative images or expressed as the median (IQR) of individual groups ( $\mathrm{n}=8$ per group). A, The representative images. B, Quantitative analysis. ( sham group, $\square$ LIRI group, $\triangle$ EPC group, $\nabla$ and l-ornithine-pretreated EPC $[E P C / L]$ group.) The upper and lower borders of the box represent the standard deviation. The middle horizontal line represents the mean. $p$-AKT, Phosphorylated protein kinase $\mathrm{B} ; p$-eNOS, phosphorylated eNOS; $p$-MLC, phosphorylated myosin light chain.

the mechanism of EPCs on eNOS, we detected the phosphorylation of AKT. The result indicated that LIRI promoted the phosphorylation of AKT, and was enhanced by the EPCs. ${ }^{28}$ However, the enhancement of EPCs on phosphorylation of AKT was partially reversed by L-NIO. Furthermore, after lung endothelial and epithelial injury, MLC is activated and phosphorylated. ${ }^{29}$ pMLC can disrupt the pulmonary endothelial barrier and enhance lung edema during the process of acute lung injury and LIRI. ${ }^{30,31}$ In this study, we found that EPC treatment significantly decreased the levels of pMLC in lung grafts, which may reflect vascular endothelial repair and replacement by EPCs. ${ }^{32}$ Therefore, the inhibition of MLC phosphorylation may be the main mechanism for protection of EPCs on endothelial hyperpermeability.

During the process of ischemia-reperfusion injury, oxidative stress can induce inflammation by activating nuclear factor-kB signaling and promoting proinflammatory cytokine production, which is critical for the development of LIRI. ${ }^{33}$ In this study, we found that EPCs decreased the levels of serum TNF- $\alpha$, IL- $1 \beta$, IL- 6 , and
IL-8, but increased the levels of anti-inflammatory IL10 in rats, consistent with previous studies. ${ }^{6,34}$ Furthermore, EPC treatment significantly attenuated the ischemia-reperfusion-upregulated nuclear factor-kBp65 activation in the lung grafts. Therefore, EPCs effectively modulated the imbalance of pro- and anti-inflammatory cytokine responses during the early development of LIRI. Moreover, we found that EPCs significantly reduced the activity of MPO in the lung grafts of rats. Such data suggest that EPC may reduce the LIRIinduced neutrophil infiltration in the grafts because MPO is highly expressed in neutrophils and usually had been used to estimate the neutrophil counts in various inflammation states. ${ }^{35,36}$ Given that aberrant proinflammatory cytokine responses and neutrophil infiltration are pathogenic factors of LIRI, the inhibition of the proinflammatory cytokine response and possible limitation of neutrophil infiltration by EPCs should contribute to their protective effect in this model.

Inflammation and oxidative stress can led to apoptosis of lung endothelial and epithelial cells, a hallmark of 

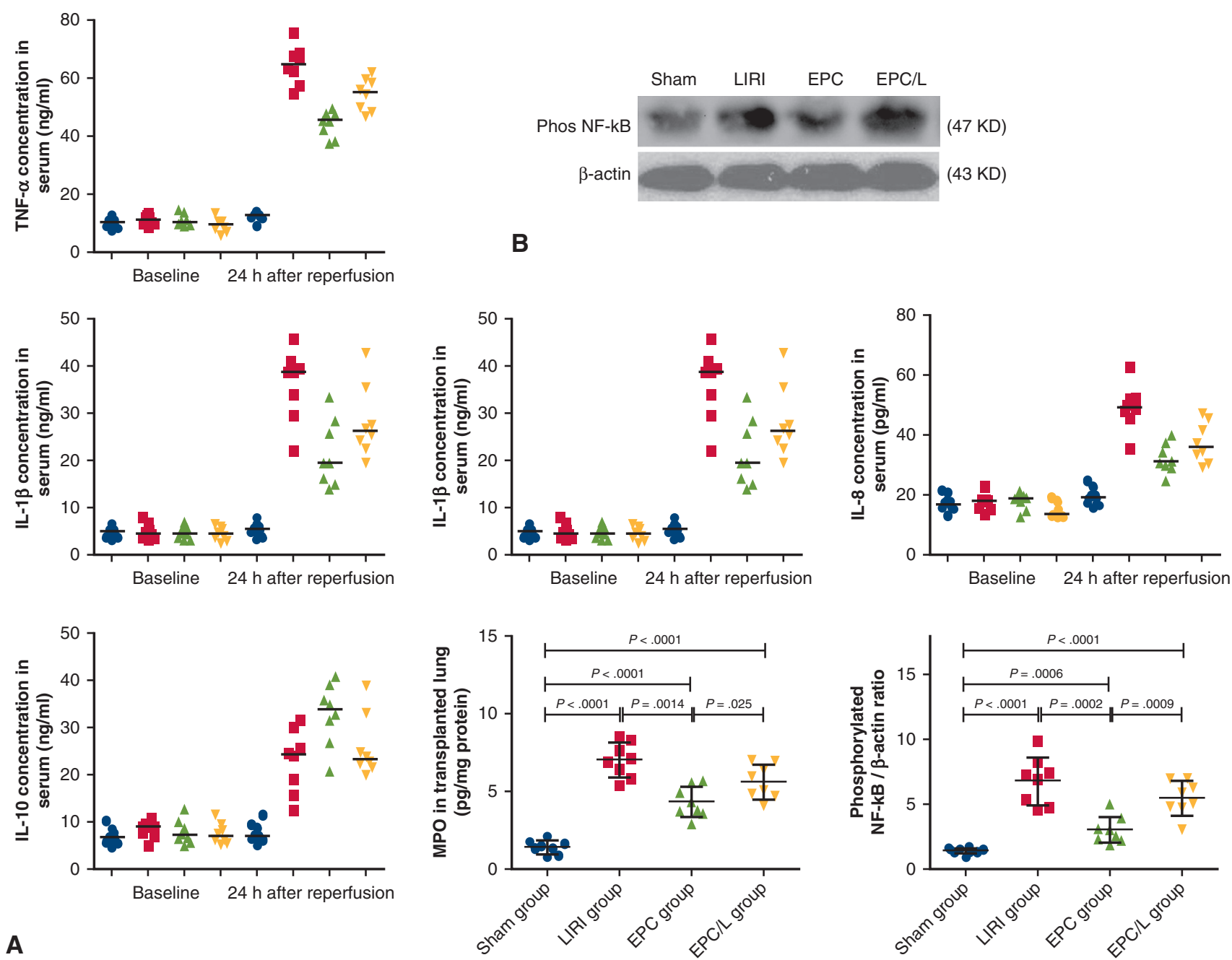

FIGURE 6. Endothelial progenitor cells $(E P C s)$ reduce the ischemia-reperfusion-induced inflammation in rats. The levels of serum tumor necrosis factor- $\alpha$ $(T N F-\alpha)$, interleukin $(I L)-1 \beta$, IL-6, IL-8, and IL-10 in individual rats were determined by enzyme-linked immunosorbent assay. The relative levels of phosphorylated nuclear factor $(N F) \mathrm{kBp} 65$ to $\beta$-actin expression in the grafted lungs of individual groups of rats were determined by Western blot. Data are representative images or expressed as the mean \pm standard deviation or median (interquartile range) of individual groups ( $\mathrm{n}=8$ per group). A, The levels of serum cytokines and lung myeloperoxidase (MPO). B, Western blot analysis of the relative levels of phosphorylated NF-kBp65 in the grafted lungs. ( sham group, $\square$ lung ischemia-reperfusion injury [LIRI] group, $\triangle \mathrm{EPC}$ group, $\nabla$ and N5-(1-iminoethyl)-1-ornithine-pretreated EPC [EPC/L] group). The upper and lower borders of the box represent the interquartile range. The middle horizontal line represents the median.

LIRI. $^{37,38}$ In this study, we found that EPC treatment significantly reduced the numbers of apoptotic cells in the lung grafts, consistent with a previous report. ${ }^{7}$ Bax and $\mathrm{Bcl}-2$ are important apoptotic regulators. Whereas Bax participates in the process of apoptosis, Bcl-2 usually has potent antiapoptotic activity to support the survival of cells. As a result, the ratio of Bax to Bcl-2 usually reflects the levels of apoptosis. ${ }^{39}$ During the process of apoptosis, proapoptotic signaling promotes the cleavage and activation of caspase 3, which leads to DNA fragmentation and cell death. Hence, the levels of cleaved caspase 3 have been widely used for evaluating the levels of apoptosis. In this study, EPC treatment significantly decreased the levels of Bax and cleaved caspase 3, but increased the levels of Bcl-2 expression in the lung grafts, which should contribute to protection against apoptosis. ${ }^{40}$ Yen and colleagues ${ }^{41}$ also reported that EPC treatment before restoration of pulmonary blood flow protected the recipients from death at 40 minutes posttransplantation in a porcine lung transplantation with extended ischemia. Given that the mobilization of EPC into the injured lung tissues needs at least 4 hours ${ }^{10}$ this short period of protection by EPCs is unlikely mediated by the biological function of EPCs. ${ }^{6,22}$ Therefore, we chose to observe the therapeutic effect of EPC on LIRI at 24 hours after lung transplantation. $^{22}$ 

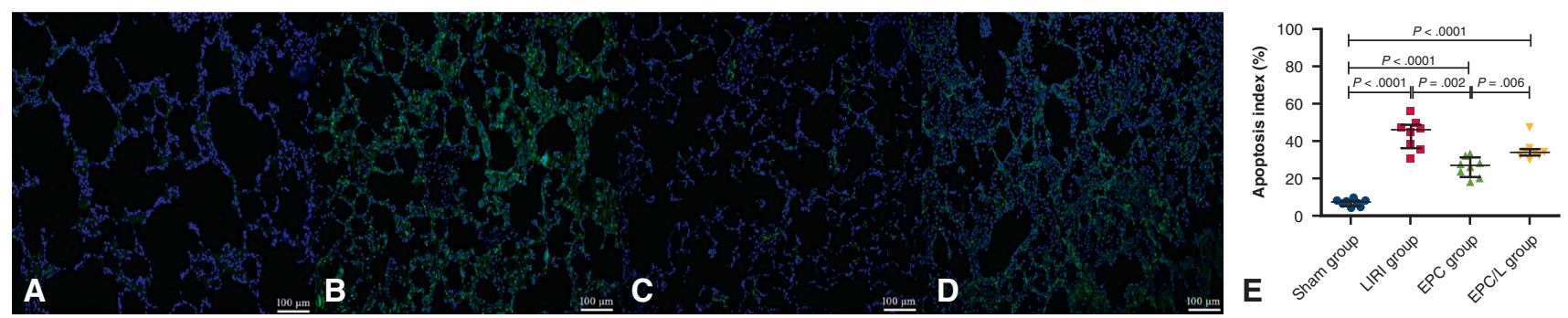

FIGURE 7. Endothelial progenitor cells $(E P C s)$ reduce the ischemia-reperfusion-induced apoptosis in the grafted lungs of rats. The cell apoptosis in the grafted lungs of individual groups of rats was determined by terminal deoxynucleotidyl transferase deoxyuridine triphosphate nick-end labeling assay (Roche Diagnostics GmbH, Science, Mannheim, Germany) assay and counterstained with 4,6-diamidino-2-phenylindole followed by examining under a fluorescent microscope. Data are representative images (magnification $\times 200$ ) or expressed as the median (interquartile range) of each group of rats ( $\mathrm{n}=8$ per group). A, The sham group. B, The lung ischemia-reperfusion injury (LIRI) group. C, EPC group. D, N5-(1-iminoethyl)-1-ornithine-pretreated EPC (EPC/L) group. E, Quantitative analysis. ( sham group, $\square$ LIRI group, $\triangle \mathrm{EPC}$ group, $\nabla$ and EPC/L group). The upper and lower borders of the box represent the standard deviation. The middle horizontal line represents the mean.

It is well known that eNOS is crucial for the survival, attachment, migration, and tube formation of EPCs. ${ }^{13,14,42}$ To investigate the mechanism of EPCs on LIRI, we administrated eNOS inhibitor-treated EPCs. The results indicated that treatment with L-NIO significantly decreased the percentages of migrated EPC in the lung grafts. Treatment with the L-NIO-treated EPCs mitigated the protective effect on the lung function, the alveoli-capillary permeability, the lung injury, and the lung cell apoptosis in this model. Such data suggest that inhibition of eNOS in EPCs may reduce the chemotaxis and recruitment of EPCs into injured lung tissue. ${ }^{13}$ Therefore, eNOS may be crucial for the therapeutic effect of EPCs on LIRI.
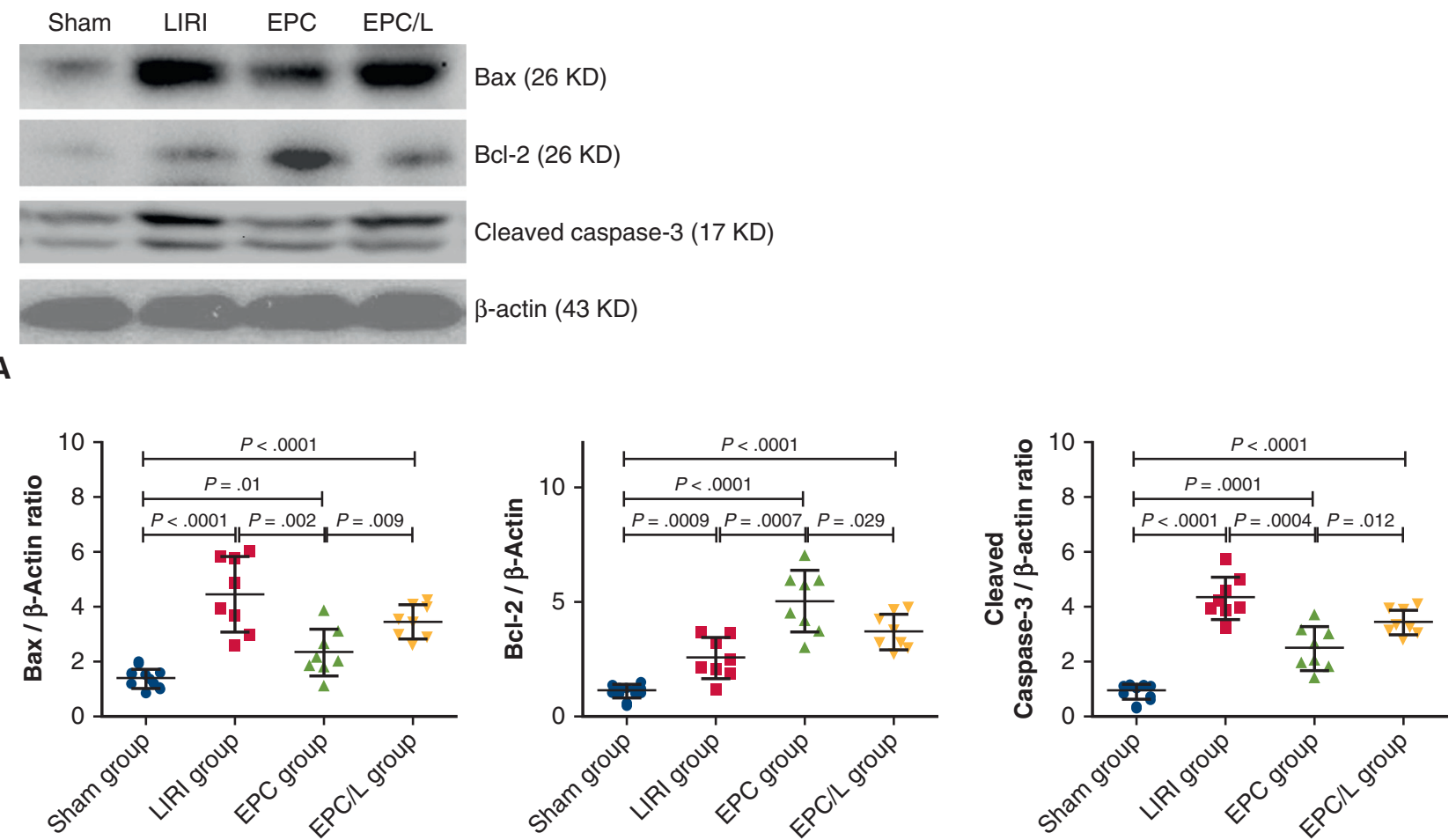

B

FIGURE 8. Endothelial progenitor cells $(E P C s)$ modulate the levels of Bax, Bcl-2, and cleaved caspase- 3 in lung grafts from rats. The relative levels of $\mathrm{Bax}, \mathrm{Bcl}-2$, and cleaved caspase- 3 to control $\beta$-actin in the grafted lung tissues of each group of rats were determined by Western blot. Data are representative images or expressed as the median (interquartile range) of each group of rats ( $\mathrm{n}=8$ per group). A, The representative images. B, Quantitative analysis of ( sham group, lung ischemia-reperfusion injury [LIRI] group, $\triangle$ EPC group, and $\nabla$ N5-(1-iminoethyl)-1-ornithine-pretreated EPC [EPC/L] group). The upper and lower borders of the box represent the standard deviation. The middle horizontal line represents the mean. 


\section{Limitations}

There are some limitations in this study. Firstly, we used a mixed population of early and advanced EPCs in this study and could not distinguish the effect of early from advanced EPCs. We are interested in investigating the exact effect of early and advanced EPCs alone on LIRI after lung transplantation in future studies. Secondly, we did not analyze whether the EPCs migrated into other organs following LIRI, although a previous observation revealed that the injected EPCs predominantly migrate into the inflammatory lung lesions following LIRI, but not other organs. ${ }^{22}$ Thirdly, during the precondition of L-NIO on EPCs, we did not observe the directly effect of L-NIO on the EPCs and whether the L-NIO can influence EPCs. Fourthly, we did not explore the role of mitogen-activated protein kinase and AKT, which are the important upstream regulatory signal transduction of eNOS in the protection EPCs on LIRI. In a future study, we will investigate the effect of EPCs preconditioned by AKT overexpression on LIRI after lung transplantation and explore the role of mitogen-activated protein kinase in the protection of EPCs.

\section{CONCLUSIONS}

The results of this study indicate that EPCs attenuated LIRI in a rat model of lung transplantation. The protective effect of EPCs against LIRI was associated with improving the structure and function of endothelium and attenuating $\mathrm{NF}-\kappa \mathrm{B}$ signaling, inflammation, and apoptosis. The protective effect of EPCs on LIRI mainly depended on the eNOS pathway.

\section{References}

1. Cottini SR, Lerch N, de Perrot M, Treggiari MM, Spiliopoulos A, Nicod L, et al. Risk factors for reperfusion injury after lung transplantation. Intensive Care Med. 2006;32:557-63.

2. Burton CM, Iversen M, Milman N, Zemtsovski M, Carlsen J, Steinbruchel D, et al. Outcome of lung transplanted patients with primary graft dysfunction. Eur J Cardiothorac Surg. 2007;31:75-82.

3. van der Kaaij NP, Kluin J, Haitsma JJ, den Bakker MA, Lambrecht BN, Lachmann B, et al. Ischemia of the lung causes extensive long-term pulmonary injury: an experimental study. Respir Res. 2008;9:28.

4. Charles EJ, Mehaffey JH, Sharma AK, Zhao Y, Stoler MH, Isbell JM, et al. Lungs donated after circulatory death and prolonged warm ischemia are transplanted successfully after enhanced ex vivo lung perfusion using adenosine A2B receptor antagonism. J Thorac Cardiovasc Surg. 2017;154: 1811-20.

5. Hwang B, Liles WC, Waworuntu R, Mulligan MS. Pretreatment with bone marrow-derived mesenchymal stromal cell-conditioned media confers pulmonary ischemic tolerance. J Thorac Cardiovasc Surg. 2016; 151:841-9.

6. Cao JP, He XY, Xu HT, Zou Z, Shi XY. Autologous transplantation of peripheral blood-derived circulating endothelial progenitor cells attenuates endotoxin-induced acute lung injury in rabbits by direct endothelial repair and indirect immunomodulation. Anesthesiology. 2012;116:1278-87.

7. Kocher AA, Schuster MD, Szabolcs MJ, Takuma S, Burkhoff D, Wang J, et al. Neovascularization of ischemic myocardium by human bone-marrow-derived angioblasts prevents cardiomyocyte apoptosis, reduces remodeling and improves cardiac function. Nat Med. 2001;7:430-6.

8. Kaushal S, Amiel GE, Guleserian KJ, Shapira OM, Perry T, Sutherland FW, et al. Functional small-diameter neovessels created using endothelial progenitor cells expanded ex vivo. Nat Med. 2001;7:1035-40.
9. Mao M, Wang SN, Lv XJ, Wang Y, Xu JC. Intravenous delivery of bone marrow-derived endothelial progenitor cells improves survival and attenuates lipopolysaccharide-induced lung injury in rats. Shock. 2010;34: 196-204.

10. Yamada M, Kubo H, Kobayashi S, Ishizawa K, Numasaki M, Ueda S, et al. Bone marrow-derived progenitor cells are important for lung repair after lipopolysaccharide-induced lung injury. J Immunol. 2004;172:1266-72.

11. Aggarwal S, Pittenger MF. Human mesenchymal stem cells modulate allogeneic immune cell responses. Blood. 2005;105:1815-22.

12. Dong L, Kang L, Ding L, Chen Q, Bai J, Gu R, et al. Insulin modulates ischemiainduced endothelial progenitor cell mobilization and neovascularization in diabetic mice. Microvasc Res. 2011;82:227-36.

13. Qiu FY, Song XX, Zheng H, Zhao YB, Fu GS. Thymosin beta4 induces endothelial progenitor cell migration via PI3K/Akt/eNOS signal transduction pathway. $J$ Cardiovasc Pharmacol. 2009;53:209-14.

14. Fu M, Li Z, Tan T, Guo W, Xie N, Liu Q, et al. Akt/eNOS signaling pathway mediates inhibition of endothelial progenitor cells by palmitate-induced ceramide. Am J Physiol Heart Circ Physiol. 2015;308:H11-7.

15. Gao W, Liu D, Li D, Che X, Cui G. Effects of hypercapnia on T cells in lung ischemia/reperfusion injury after lung transplantation. Exp Biol Med (Maywood). 2014;239:1597-605

16. Gielis JF, Quirynen L, Briede JJ, Roelant E, Cos P, Van Schil PEY. Pathogenetic role of endothelial nitric oxide synthase uncoupling during lung ischaemiareperfusion injury. Eur J Cardiothorac Surg. 2017;52:256-63.

17. Asahara T, Murohara T, Sullivan A, Silver M, van der Zee R, Li T, et al. Isolation of putative progenitor endothelial cells for angiogenesis. Science. 1997;275: 964-7.

18. Hur J, Yoon CH, Kim HS, Choi JH, Kang HJ, Hwang KK, et al. Characterization of two types of endothelial progenitor cells and their different contributions to neovasculogenesis. Arterioscler Thromb Vasc Biol. 2004;24:288-93.

19. Rehman J, Li J, Orschell CM, March KL. Peripheral blood "endothelial progenitor cells" are derived from monocyte/macrophages and secrete angiogenic growth factors. Circulation. 2003;107:1164-9.

20. Burger D, Vinas JL, Akbari S, Dehak H, Knoll W, Gutsol A, et al. Human endothelial colony-forming cells protect against acute kidney injury: role of exosomes. Am J Pathol. 2015;185:2309-23.

21. Li S, Tian Y, Huang X, Zhang Y, Wang D, Wei H, et al. Intravenous transfusion of endothelial colony-forming cells attenuates vascular degeneration after cerebral aneurysm induction. Brain Res. 2014;1593:65-75.

22. Kahler CM, Wechselberger J, Hilbe W, Gschwendtner A, Colleselli D Niederegger $\mathrm{H}$, et al. Peripheral infusion of rat bone marrow derived endothelial progenitor cells leads to homing in acute lung injury. Respir Res. 2007;8:50.

23. Ng CS, Wan S, Arifi AA, Yim AP. Inflammatory response to pulmonary ischemia-reperfusion injury. Surg Today. 2006;36:205-14.

24. Okada M, Yamashita C, Okada M, Okada K. Contribution of endothelin-1 to warm ischemia/reperfusion injury of the rat lung. Am J Respir Crit Care Med. 1995; 152:2105-10.

25. Shaw MJ, Shennib H, Bousette N, Ohlstein EH, Giaid A. Effect of endothelin receptor antagonist on lung allograft apoptosis and NOSII expression. Ann Thorac Surg. 2001;72:386-90.

26. Ovechkin AV, Lominadze D, Sedoris KC, Gozal E, Robinson TW, Roberts AM. Inhibition of inducible nitric oxide synthase attenuates platelet adhesion in subpleural arterioles caused by lung ischemia-reperfusion in rabbits. J Appl Physiol (1985). 2005;99:2423-32.

27. Kaminski A, Kasch C, Zhang L, Kumar S, Sponholz C, Choi YH, et al. Endothelial nitric oxide synthase mediates protective effects of hypoxic preconditioning in lungs. Respir Physiol Neurobiol. 2007;155:280-5.

28. Vinas JL, Burger D, Zimpelmann J, Haneef R, Knoll W, Campbell P, et al Transfer of microRNA-486-5p from human endothelial colony forming cell-derived exosomes reduces ischemic kidney injury. Kidney Int. 2016;90: 1238-50.

29. Muller HC, Witzenrath M, Tschernig T, Gutbier B, Hippenstiel S, Santel A, et al. Adrenomedullin attenuates ventilator-induced lung injury in mice. Thorax. 2010; 65:1077-84

30. Zhang HF, Li TB, Liu B, Lou Z, Zhang JJ, Peng JJ, et al. Inhibition of myosin light chain kinase reduces NADPH oxidase-mediated oxidative injury in rat brain following cerebral ischemia/reperfusion. Naunyn Schmiedebergs Arch Pharmacol. 2015;388:953-63.

31. Rossi JL, Velentza AV, Steinhorn DM, Watterson DM, Wainwright MS MLCK210 gene knockout or kinase inhibition preserves lung function following 
endotoxin-induced lung injury in mice. Am J Physiol Lung Cell Mol Physiol. 2007;292:L1327-34.

32. Werner N, Kosiol S, Schiegl T, Ahlers P, Walenta K, Link A, et al. Circulating endothelial progenitor cells and cardiovascular outcomes. N Engl J Med. 2005; 353:999-1007.

33. Ross SD, Kron IL, Gangemi JJ, Shockey KS, Stoler M, Kern JA, et al. Attenuation of lung reperfusion injury after transplantation using an inhibitor of nuclear factor-kappaB. Am J Physiol Lung Cell Mol Physiol. 2000;279: L528-36.

34. Zhang R, Yang J, Yuan J, Song B, Wang Y, Xu Y. The therapeutic value of bone marrow-derived endothelial progenitor cell transplantation after intracerebral hemorrhage in rats. Front Neurol. 2017;8:174.

35. Krawisz JE, Sharon P, Stenson WF. Quantitative assay for acute intestinal inflammation based on myeloperoxidase activity. Assessment of inflammation in rat and hamster models. Gastroenterology. 1984;87:1344-50.

36. Andonegui G, Bonder CS, Green F, Mullaly SC, Zbytnuik L, Raharjo E, et al. Endothelium-derived Toll-like receptor-4 is the key molecule in LPS-induced neutrophil sequestration into lungs. J Clin Invest. 2003;111:1011-20.

37. Rivo J, Zeira E, Galun E, Matot I. Activation of A3 adenosine receptor provides lung protection against ischemia-reperfusion injury associated with reduction in apoptosis. Am J Transplant. 2004;4:1941-8.
38. Rivo J, Zeira E, Galun E, Einav S, Linden J, Matot I. Attenuation of reperfusion lung injury and apoptosis by A2A adenosine receptor activation is associated with modulation of Bcl-2 and Bax expression and activation of extracellular signal-regulated kinases. Shock. 2007;27:266-73.

39. Cartron PF, Juin P, Oliver L, Meflah K, Vallette FM. Impact of proapoptotic proteins Bax and Bak in tumor progression and response to treatment. Expert Rev Anticancer Ther. 2003;3:563-70.

40. Qiu J, Li W, Feng S, Wang M, He Z. Transplantation of bone marrow-derived endothelial progenitor cells attenuates cerebral ischemia and reperfusion injury by inhibiting neuronal apoptosis, oxidative stress and nuclear factor-kappaB expression. Int J Mol Med. 2013;31:91-8.

41. Yen YT, Roan JN, Fang SY, Chang SW, Tseng YL, Lam CF. Autologous endothelial progenitor cells improve allograft survival in porcine lung transplantation with prolonged ischemia. Ann Transl Med. 2016;4:277.

42. Hoffmann J, Haendeler J, Aicher A, Rossig L, Vasa M, Zeiher AM, et al. Aging enhances the sensitivity of endothelial cells toward apoptotic stimuli: important role of nitric oxide. Circ Res. 2001;89:709-15.

Key Words: endothelial progenitor cells, lung transplantation, ischemia/reperfusion injury, inflammation 